\title{
Joint Automatic Equalization \\ for Data Communication
}

Robert W. Chang

Bell Telephone Laboratories, Incorporated Holmdel, New Jersey

\begin{abstract}
$\underline{\text { ABSTRACT }}$
In data transmission systems with transversal filter equalization, the adjustment of the demodulating carrier phase, the receiver sampling time, and the equalizer tap gains are critical to the system's performance. These parameters cannot be set optimally by independent adjustments. In this talk, a method of jointly setting these parameters based on a minimum mean-square error equalization criterion is described. The possible implementation of the method and its applications to data communication are discussed. Computer simulations show that, when compared with systems using independent settings, the method proposed may offer a reduction in mean-square equalization error of as much as $10 \mathrm{db}$.
\end{abstract}




\section{Joint Automatic Equalization \\ for Data Communication}

Robert W. Chang

Bell Telephone Laboratories, Incorporated

Holmdel, New Jersey

\section{Introduction}

The subject of automatic equalization with transversal equalizer has been extensively studied. However, previous studies have not considered the problem of setting the receiver sampling time or the demodulating carrier phase jointly with the tap gains of the transversal equalizer. Using minimum mean-square error as the performance criterion, a method of jointly setting these parameters is developed. The method will improve the parameter settings obtained from independent adjustments.

A possible simple implementation of the method is described. The application to data communication is illustrated in Section 2.2.

II. Mathematical Model and Applications

The mathematical model and the performance criterion will be formulated in general terms in Section 2.1. They will be illustrated in Section 2.2 by considering applications to data transmission. 


\section{I A General Formulation}

As shown in Figure 1, we consider amplitude modulation systems with coherent detection. The system may be singlesideband, vestigial-sideband or double-sideband. The results apply to baseband systems by setting the demodulating carrier frequency $f_{c}$ and phase $\theta$ to zero.

When an impulse $\delta(t)$ is applied to the transmitter input, a signal $a(t)$ is received at the receiving filter output. The Fourier transform of $a(t)$ is denoted by $A(f)$. (The Fourier transform of a function will be consistently denoted by the appropriate capital letter.) We assume that $a(t)$ is bandlimited, i.e.,

$$
A(f) \neq 0 \text {, only for } f_{1}<|f|<f_{2} \text {. }
$$

The signal $a(t)$ is demodulated as shown in Figure 1 . The carrier frequency $f_{c}$ can be recovered at the receiver from transmitted pilot carriers (a general practice in data transmission). We shall consider the setting of the demodulating carrier phase $\theta$ which is critical to the system performance.

The demodulator output is a signal $\gamma(t)$ with Fourier transform $\Gamma(f)$. Note that $\gamma(t)$ is band-limited because $a(t)$ is. Therefore, we may assume

$$
\Gamma(f)=0 \quad|f|>f_{0} .
$$


The transfer function of the $(2 \mathrm{~N}+\mathrm{I})$ - tap transversal equalizer with tap gains $e_{n}, n=-N$ to $N$ spaced at $T_{0}-$ second intervals is denoted $E(f)$.

When an impulse $\delta(t)$ is applied to the transmitter input, the transversal equalizer output is $s(t)$. Note that $s(t)$ is the overall impulse response of the system. In designing such systems, one frequently encounters the following requirement (an illustrative example will be given in Section 2.2):

Design the system such that the impulse response $s(t)$ of the system matches a prescribed impulse response $q(t)$, but an arbitrary time shift $t_{0}$ can be allowed in $q(t)$.

The time shift $t_{0}$ is allowed because it makes no difference whether $s(t)$ matches $q(t)$ or $q\left(t-t_{0}\right)$, as long as $t_{0}$ is known.

In order to carry out the design, we must choose a performance criterion. In this study, the familiar meansquare error criterion is chosen, i.e., we shall design the receiver to minimize the quantity

$$
\varepsilon=\int_{-\infty}^{\infty}\left[s(t)-q\left(t-t_{0}\right)\right]^{2} d t
$$

Since $q\left(t-t_{0}\right)$ depends on $t_{0}$ and since $s(t)$ depends on the demodulating carrier phase $\theta$ and the equalizer tap gains $\left\{e_{n}\right\}$, $\mathcal{E}$ is a function of $t_{0}, \theta$, and $\left\{e_{n}\right\}$. Let us denote the value 
of $t_{0}, \theta$, and $\left\{e_{n}\right\}$ which minimize $\varepsilon$ as, respectively, $t_{0}^{*}, \theta^{*}$, and $\left\{e_{n}^{*}\right\}$. As can be seen in later derivations, $t_{0}^{*}$ depends on $\theta$ and $\left\{e_{n}\right\}, \theta^{*}$ depends on $t_{0}$ and $\left\{e_{n}\right\}$, and $\left\{e_{n}^{*}\right\}$ depends on $t_{0}$ and $\theta$. Therefore, it is not possible to set these parameters optimally by independent adjustments. We must adjust $t_{0}, \theta$, and $\left\{e_{n}\right\}$ jointly in order to minimize $\mathcal{E}$.

The above brings out an interesting point. It is shown that in order to minimize $\varepsilon$ we must select $t_{0}$. Thus, the so called arbitrary time shift in the original requirement is really not arbitrary. The freedom of choosing $t_{0}$ must be utilized in the system design.

In order to equalize the system over the frequency band $|f| \leq f_{0}$, we set the equalizer tap spacing to

$$
T_{0}=\frac{1}{2 f_{0}} \quad \text { seconds. }
$$

As can be seen in the application in the next section, $t_{0}$ has the physical meaning of receiver sampling time in data transmission systems. Therefore, we refer to $t_{0}$ as sampling time.

\subsection{Application to Data Transmission System}

The system considered in Figure 1 may be a singlesideband data transmission system transmitting Class IV partial response signals. ${ }^{3}$ It is well known that in such a system the information symbols are precoded and then transmitted at the Nyquist rate. At the receiver the transversal equalizer output 
is fed to a sampler and is sampled at the Nyquist rate to recover the information symbols. Let the sampling instants be denoted by

$$
t_{0}+k T_{0}, \quad k=-\infty \text { to } \infty .
$$

As shown in Figure 2 of Reference 3, the system operates in the desired manner if the overall impulse response $s(t)$ has the following values

$$
\ldots 0,0,1,0,-1,0,0, \ldots
$$

at the sampling instants. These desired values may be denoted by $\left\{q_{k}\right\}$, where

$$
\begin{aligned}
q_{k} & =1, & & k=-1 \\
& =-1, & & k=1 \\
& =0, & & \text { all other } k .
\end{aligned}
$$

The true time samples of $s(t)$ are

$$
\mathrm{s}_{\mathrm{k}} \triangleq \mathrm{s}\left(\mathrm{t}_{\mathrm{O}}+\mathrm{kT} \mathrm{T}_{\mathrm{o}}\right), \quad \mathrm{k}=-\infty \text { to } \infty
$$

These true time samples are denoted by $\left\{s_{k}\right\}$. Desired operation is achieved if $s_{k}=q_{k}$ for all $k$. In practice it is not possible to achieve this, but the difference between $s_{k}$ and $q_{k}$ can be minimized by setting $\theta, t_{0}$, and $\left\{e_{n}\right\}$. A proper criterion 
is the minimization of the error

$$
\varepsilon_{0}=\sum_{k=-\infty}^{\infty}\left(s_{k}-q_{k}\right)^{2}
$$

By the sampling theorem, (6) can be rewritten as

$$
\varepsilon_{0}=\frac{1}{\mathrm{~T}_{0}} \int_{-\infty}^{\infty}\left[s(t)-\mathrm{q}\left(t-t_{0}\right)\right]^{2} d t
$$

where $q(t)$ is defined by

$$
\mathrm{q}\left(\mathrm{kT} \mathrm{T}_{0}\right)=\mathrm{q}_{\mathrm{k}}, \quad \mathrm{k}=-\infty \text { to } \infty \text {. }
$$

Comparing (7) with (3) shows that this is exactly the same problem formulated in Section 2.1.

For illustration purposes, we have chosen to consider a data transmission system transmitting Class IV partial response signals. But clearly the method applies to all partial response systems. (One needs only to change $q(t)$ according to the type of partial response signal transmitted.) III. Single-Sideband Systems

For clarity, we shall consider single-sideband systems in this section and vestigial- and double-sideband systems in Section IV. 


\subsection{Analysis}

In single-sideband systems, we have

$$
f_{c} \leq f_{1}
$$

or

$$
\mathrm{f}_{\mathrm{c}} \geq \mathrm{f}_{2}
$$

In analyzing carrier signals, it is most convenient to use Hilbert transform techniques. As is well known, the demodulator output $\gamma(t)$ is related to the input a(t) by

$$
\gamma(t)=\cos \left(2 \pi f_{c} t+\theta\right) a(t)+\sin \left(2 \pi f_{c} t+\theta\right) \hat{a}(t)
$$

where $\hat{a}(t)$ is the Hilbert transform of $a(t)$. Since it is impractical to use the standard sign "^" to denote Hilbert transform when dealing with lengthy time functions, we shall sometimes use the $\operatorname{sign} H, i . e ., H[a(t)]=\hat{a}(t)$.

It is seen from Figure 1 that

$$
s(t)=\sum_{n=-N}^{N} e_{n} \gamma\left(t-n T_{0}-N T_{0}\right)
$$

We shall consider setting $\theta, t_{0}$, and $\left\{e_{n}\right\}$ jointly by a method of steepest descent; therefore, we evaluate the partial derivatives $\partial \varepsilon / \partial \theta, \partial \varepsilon / \partial t_{0}$, and $\partial \varepsilon / \partial e_{n}$. 


$$
\begin{aligned}
& \text { Since } q\left(t-t_{0}\right) \text { is independent of } \theta \text {, we } \\
& \frac{\partial \varepsilon}{\partial \theta}=\int_{-\infty}^{\infty} 2\left[s(t)-q\left(t-t_{0}\right)\right] \frac{\partial s(t)}{\partial \theta} d t .
\end{aligned}
$$

In writing (13), we have exchanged the order of differentiation and integration (the underlying conditions for making such an exchange are easily satisfied by $s(t)$ and $q(t)$ encountered in transmission systems).

Substituting (II) and (I2) and taking the partial derivative gives

$$
\begin{aligned}
\frac{\partial s(t)}{\partial \theta}=\sum_{n=-N}^{N} e_{n}\{ & -\sin \left[2 \pi f_{c}\left(t-n T_{0}-N T_{0}\right)+\theta\right] a\left(t-n T_{0}-N T_{0}\right) \\
& \left.+\cos \left[2 \pi f_{c}\left(t-n T_{0}-N T_{0}\right)+\theta\right] \hat{a}\left(t-n T_{0}-N T_{0}\right)\right\} .
\end{aligned}
$$

From (12) and (11),

$$
\begin{aligned}
\hat{s}(t)= & \sum_{n=-N}^{N} e_{n} \hat{\gamma}\left(t-n T_{0}-N T_{0}\right) \\
= & \sum_{n=-N}^{N} e_{n}\left[H\left\{\cos \left[2 \pi f_{c}\left(t-n T_{0}-N T_{0}\right)+\theta\right] a\left(t-n T_{0}-N T_{0}\right)\right\}\right. \\
& \left.+H\left\{\sin \left[2 \pi f_{c}\left(t-n T_{0}-N T_{0}\right)+\theta\right] \hat{a}\left(t-n T_{0}-N T_{0}\right)\right\}\right] .
\end{aligned}
$$


In single-sideband modulation, we have either (9) or (10). Let us consider (9) first. When (9) holds, the frequency spectrum of $a(t), A(f)$, does not overlap the spectra of $\cos 2 \pi f_{c} t$ and sin $2 \pi f_{c} t$. Furthermore, $A(f)$ occupies a higher frequency band; therefore,

$$
H\left[\cos \left(2 \pi f_{c} t+\theta\right) a(t)\right]=\cos (2 \pi f c t+\theta) \hat{a}(t)
$$

and

$$
\begin{aligned}
H\left[\sin \left(2 \pi f_{c} t+\theta\right) \hat{a}(t)\right] & =-\sin \left(2 \pi f_{c} t+\theta\right) a(t) \\
f_{c} & \leq f_{I} .
\end{aligned}
$$

Substituting (16) into (15) gives

$$
\begin{array}{r}
\hat{s}(t)=\sum_{n=-N}^{N} e_{n}\left\{\cos \left[2 \pi f_{c}\left(t-n T_{0}-N T_{0}\right)+\theta\right] \hat{a}\left(t-n T_{0}-N T_{0}\right)\right. \\
\left.-\sin \left[2 \pi f_{c}\left(t-n T_{0}-N T_{0}\right)+\theta\right] a\left(t-n T_{0}-N T_{0}\right)\right\} \\
f_{c} \leq f_{I} .
\end{array}
$$

Comparing (17) with (14) shows that

$$
\hat{s}(t)=\frac{\partial s(t)}{\partial \theta}, \quad f_{c} \leq f_{I} .
$$

Substituting (18) into (13) gives

$$
\frac{\partial \varepsilon_{I}}{\partial \theta}=\int_{-\infty}^{\infty} 2\left[s(t)-q\left(t-t_{0}\right)\right] \hat{s}(t) d t, \quad f_{c} \leq f_{I} .
$$


Since a function and its Hilbert transform are orthogonal, (19) reduces to

$$
\frac{\partial \varepsilon}{\partial \theta}=-2 \int_{-\infty}^{\infty} q\left(t-t_{0}\right) \hat{s}(t) d t \quad f_{c} \leq f_{I} .
$$

Thus, $\frac{\partial \varepsilon}{\partial \theta}$ can be generated by correlating $q\left(t-t_{0}\right)$ with $\hat{s}(t)$. Note that the transversal equalizer output will be $\hat{s}(t)$ instead of $s(t)$ if the demodulating carrier $\cos \left(2 \pi f_{c} t+\theta\right)$ is replaced by $\cos \left[2 \pi f_{c} t+\theta+\pi / 2\right]$. However, even though $\hat{s}(t)$ can be generated in this simple fashion, we prefer not to generate $\hat{s}(t)$ because the system must be used instead to generate $s(t)$ to compute the other partial derivatives $\partial \mathcal{E} / \partial t_{0}$ and $\partial \mathcal{E} / \partial \mathrm{e}_{\mathrm{n}}$. Therefore, we change (20) into the form

$$
\frac{\partial E}{\partial \theta}=2 \int_{-\infty}^{\infty} \hat{q}\left(t-t_{0}\right) s(t) d t, \quad f_{c} \leq f_{I} .
$$

This step can be easily verified by Parseval's theorem. Now we need only to correlate $s(t)$ with an easily generated $\hat{\mathrm{q}}\left(\mathrm{t}-\mathrm{t}_{0}\right)$ to obtain $\partial \varepsilon / \partial \theta$.

The above is for the case $f_{c} \leq f_{I}$. In the other case, $f_{c} \geq f_{2}$, the frequency spectrum of $a(t)$ occupies a frequency band lower than $f_{c}$; therefore, (16) should be rewritten as 


$$
\begin{aligned}
& H\left[\cos \left(2 \pi f_{c} t+\theta\right) a(t)\right]=\sin \left(2 \pi f_{c} t+\theta\right) a(t) \\
& H\left[\sin \left(2 \pi f_{c} t+\theta\right) \hat{a}(t)\right]=-\cos \left(2 \pi f_{c} t+\theta\right) \hat{a}(t) \\
& f_{c} \geq f_{2} .
\end{aligned}
$$

Repeating the steps from (17) to (21), we get

$$
\frac{\partial \mathcal{E}}{\partial \theta}=-2 \int_{-\infty}^{\infty} s(t) \hat{q}\left(t-t_{0}\right) d t, \quad f_{c} \geq f_{2}
$$

Note from (23) and (21) that the sign of the correlator output must be reversed when one shifts the carrier frequency from one side of $A(f)$ to the other side.

By noting that $s(t)$ is independent of $t_{0}$ and that the integration of $q^{2}\left(t-t_{0}\right)$ from $-\infty$ to $\infty$ is independent of $t_{0}$, one obtains

$$
\begin{aligned}
\frac{\partial \mathcal{E}}{\partial t_{0}} & =-2 \int_{-\infty}^{\infty} s(t)\left[\frac{\partial}{\partial t_{0}} q\left(t-t_{0}\right)\right] d t \\
& =2 \int_{-\infty}^{\infty} s(t)\left[\frac{\partial}{\partial t} q\left(t-t_{0}\right)\right] d t .
\end{aligned}
$$


Thus, $\partial \varepsilon / \partial t_{0}$ can be generated by correlating $s(t)$ with an easily generated signal $\frac{\partial}{\partial t} q\left(t-t_{0}\right)$.

Finally, from (3) and (12)

$$
\begin{aligned}
\frac{\partial E}{\partial e_{n}} & =\int_{-\infty}^{\infty} 2\left[s(t)-q\left(t-t_{0}\right)\right] \frac{\partial s(t)}{\partial e_{n}} d t \\
& =\int_{-\infty}^{\infty} 2\left[s(t)-q\left(t-t_{0}\right)\right] \gamma\left(t-n T_{0}-N T_{0}\right) d t \\
n & =-N \text { to } N .
\end{aligned}
$$

Thus, $\frac{\partial E}{\partial e_{n}}$ can be generated by correlating the error signal $\left[s(t)-q\left(t-t_{0}\right)\right]$ with the output $\gamma\left(t-n T_{0}-N T_{0}\right)$ of the nth tap. This is similar to the concept in Reference 2 where the problem of setting the tap gains $\left\{e_{n}\right\}$ was considered.

Finally, it can be seen by setting (25) to zero that the optimum value of $e_{n}, e_{n}^{*}$, depends on $\theta$ and $t_{0}$. Similarly, it can be seen ${ }^{\dagger}$ from (2I) or (23) that $\theta^{*}$ depends on $t_{0}$ and

F For instance, setting (2l) to zero gives

$$
\begin{aligned}
& \sum_{n=-N}^{N} e_{n}^{\infty} \hat{q}\left(t-t_{0}\right)\left\{\cos \left[2 \pi f_{c}\left(t-n T_{0}-N T_{0}\right)+\theta\right] a\left(t-n T_{0}-N T_{0}\right)\right. \\
& \left.+\sin \left[2 \pi f_{c}\left(t-n T_{0}-N T_{0}\right)+\theta\right] \hat{a}\left(t-n T_{0}-N T_{0}\right)\right\} d t=0 \\
& \text { If } f_{c} \leq f_{1}, \theta^{*} \text { must be a solution of the above equation. It } \\
& \text { can be easily seen that } \theta^{*} \text { is a function of } t_{0} \text { and }\left\{e_{n}\right\} \text {. }
\end{aligned}
$$


$\left\{e_{n}\right\}$, and from (24) that $t_{0}^{*}$ depends on $\theta$ and $\left\{e_{n}\right\}$. Therefore, it is not possible to set these parameters optimally by independent adjustments. 3.2 A Method of Setting $\theta, t_{0}$, and $\left\{e_{n}\right\}$ Jointly It is seen from (21), (23), (24), and (25) that the partial derivatives can be simultaneously generated by simple means (details to be described shortly). Based on this, the following method of setting $\theta, t_{0}$, and $\left\{e_{n}\right\}$ jointly is proposed. For brevity, this method will be called the joint method.

In the joint method, the parameters $\theta, t_{0}$, and $\left\{e_{n}\right\}$ are set jointly in a training period prior to data transmission. In the training period, isolated test pulses are transmitted. For instance, $\delta(t)$ in Figure 1 may be one of the test pulses. The transmission of $\delta(t)$ generates a signal $s(t)$ at the equalizer output. From $s(t)$ the partial derivatives $\partial \varepsilon / \partial \theta, \partial \varepsilon / \partial t_{0}$, and $\partial \varepsilon / \partial e_{n}$ are computed whose signs indicate in which direction each of the parameters should be changed in order to minimize $\varepsilon$. After the changes are all made, another test pulse is transmitted and the process is repeated. When all partial derivatives become zero, the parameters are locked and the training period terminated. Clearly the joint method will minimize $\mathcal{E}$ if the parameters are initially set within the convex region of the 
absolute minimum of $\varepsilon$. Otherwise a relative minimum of $\varepsilon$ may be obtained. However, in either case the joint method proposed improves the initial parameter settings.

A block diagram of the joint method is shown in

Figure 2. The partial derivatives $\partial \varepsilon / \partial e_{n}, \partial \varepsilon / \partial t_{0}$, and $\partial \mathcal{E} / \partial \theta$ are computed, respectively, according to (25), (24), and (23). (We assume $f_{c} \geq f_{2}$. If $f_{c} \leq f_{1}$, the correlator output w1Il be - $\partial \mathfrak{E} / \partial \theta$ instead of $\partial \mathfrak{E} / \partial \theta)$.

As shown in Figure 2, $\partial \mathcal{E} / \partial e_{n}$ is obtalned by correlating the output of the nth tap with the error signal. The same network in Reference 2 can be used. Note that an additional $2 \mathrm{~N}$ such networks (not shown in Figure 2) are required for computing $\partial \varepsilon / \partial e_{n}$ for adjusting the other $2 N$ taps. These $2 \mathrm{~N}+1$ networks are required for adjusting the equalizer regardless of whether $t_{0}$ and $\theta$ are to be set jointly with $\left\{e_{n}\right\}$. The partial derivatives $\partial \mathcal{E} / \partial t_{0}$ and $\partial \mathcal{E} / \partial \theta$ can be computed simultaneously with $\partial \mathcal{E} / \partial e_{n}$ as shown in Figure 2. The differentiator used may be implemented with an RC circuit. It is not difficult to implement the $90^{\circ}$ phase shifter because $Q(f)$, the Fourier transform of $q(t)$, is band-limited. (The phase shifter may have arbitrary amplitude and phase for $\left.|f|>f_{0} \cdot\right)$ The correlators can be implemented using the network described in Reference 2. Thus, with a few added networks, $\partial E / \partial t_{0}$ and $\partial E / \partial \theta$ can be computed simultaneously with $\partial \varepsilon / \partial e_{n}$, and $t_{0}$ and $\theta$ can be set jointly with the equalizer. 
Like any other method, the details of the above method can be easily modified in various ways. In order to avoid a tedious discussion and because the same principle is utilized, the modifications are not discussed here.

IV. Vestigial-and Double-Sideband Systems

For vestigial- and double-sideband systems,

$$
\mathrm{f}_{1}<\mathrm{f}_{c}<\mathrm{f}_{2}
$$

The partial derivatives $\partial \mathcal{E} / \partial t_{0}$ and $\partial \varepsilon / \partial e_{n}$ are again given by (24) and (25). However, $\partial \mathcal{E} / \partial \theta$ will take the form

$$
\frac{\partial \varepsilon}{\partial \theta}=\int_{-\infty}^{\infty} 2\left[s(t)-q\left(t-t_{0}\right)\right] \tilde{s}(t) d t
$$

where $\tilde{s}(t)$ is the transversal equalizer output when the demodulating carrier $\cos \left(2 \pi \mathrm{f}_{c} t+\theta\right)$ in Figure 1 is replaced by $\cos \left(2 \pi f_{c} t+\theta+\pi / 2\right)$. Owing to $(26), \tilde{s}(t)$ is not the Hilbert transform of $s(t)$ and (27) cannot be simplified much further. Thus, for vestigial- and double-sideband systems the parameters can be jointly set by the following procedure:

1. Transmit a test puIse and use demodulating carrier $\cos \left(2 \pi f_{c} t+\theta\right)$. The equalizer output is $s(t)$. Compute $\partial \varepsilon / \partial t_{0}$ and $\partial \varepsilon / \partial e_{n}$ as shown in Figure 2. Store $\left[s(t)-q\left(t-t_{0}\right)\right]$ for the next step. 
2. Transmit the next test pulse and use demodulating carrier $\cos \left(2 \pi f_{c} t+\theta+\pi / 2\right)$. The equalizer output is $\tilde{s}(t)$. Correlate $\tilde{s}(t)$ with the previously stored $\left[s(t)-q\left(t-t_{0}\right)\right]$ to obtain $\partial \mathcal{E} / \partial \theta$.

3. Adjust $t_{0}, \theta$, and $\left\{e_{n}\right\}$ according to the signs of the partial derivatives. Then repeat the above steps.

The steps are repeated until the partial derivatives become zero. The parameters are then locked and the training period terminated.

\section{Summary and Conclusion}

It is customary in present systems to set the transversal equalizer, the demodulating carrier phase, and the recelver sampling time independently. It is shown in this study that these parameters cannot be set optimally by independent adjustments.

Based on a minimum mean-square error equalization criterion, a method of jointly setting these parameters is proposed. For single-sideband systems, the required circuitry is only slightly more complicated than that for setting the transversal equalizer alone. For vestigialsideband or double-sideband systems, a more complicated circuitry is required. Applications to data transmission (particularly to a single-sideband partial response system) are considered in Section 2.2. 


\begin{abstract}
A computer program has been written to evaluate the performance of the proposed method for data transmission over voice channels. A single sideband data transmission system using class IV partial response signal and a nine-tap transversal equalizer has been simulated. The simulation shows that, when compared with systems using independent settings, the joint method proposed may offer a reduction in mean-square equalization error of as much as $10 \mathrm{db}$.
\end{abstract}




\section{REFERENCES}

1. Rudin, H. R., Automatic Equalization Using Transversal Filters, IEEE Spectrum, January, 1967, pp. 53-59.

2. Lucky, R. W., and Rudin, H. R., An Automatic Equalizer for General - Purpose Communication Channels, B.S.T.J., 46, November, 1967, pp. 2179-2208.

3. Becker, F. K., Kretzmer, E. R., and Sheehan, J. R., A New Signal Format for Efficient Data Transmission, B.S.T.J., 45, May-June 1966, pp. 755-758. 

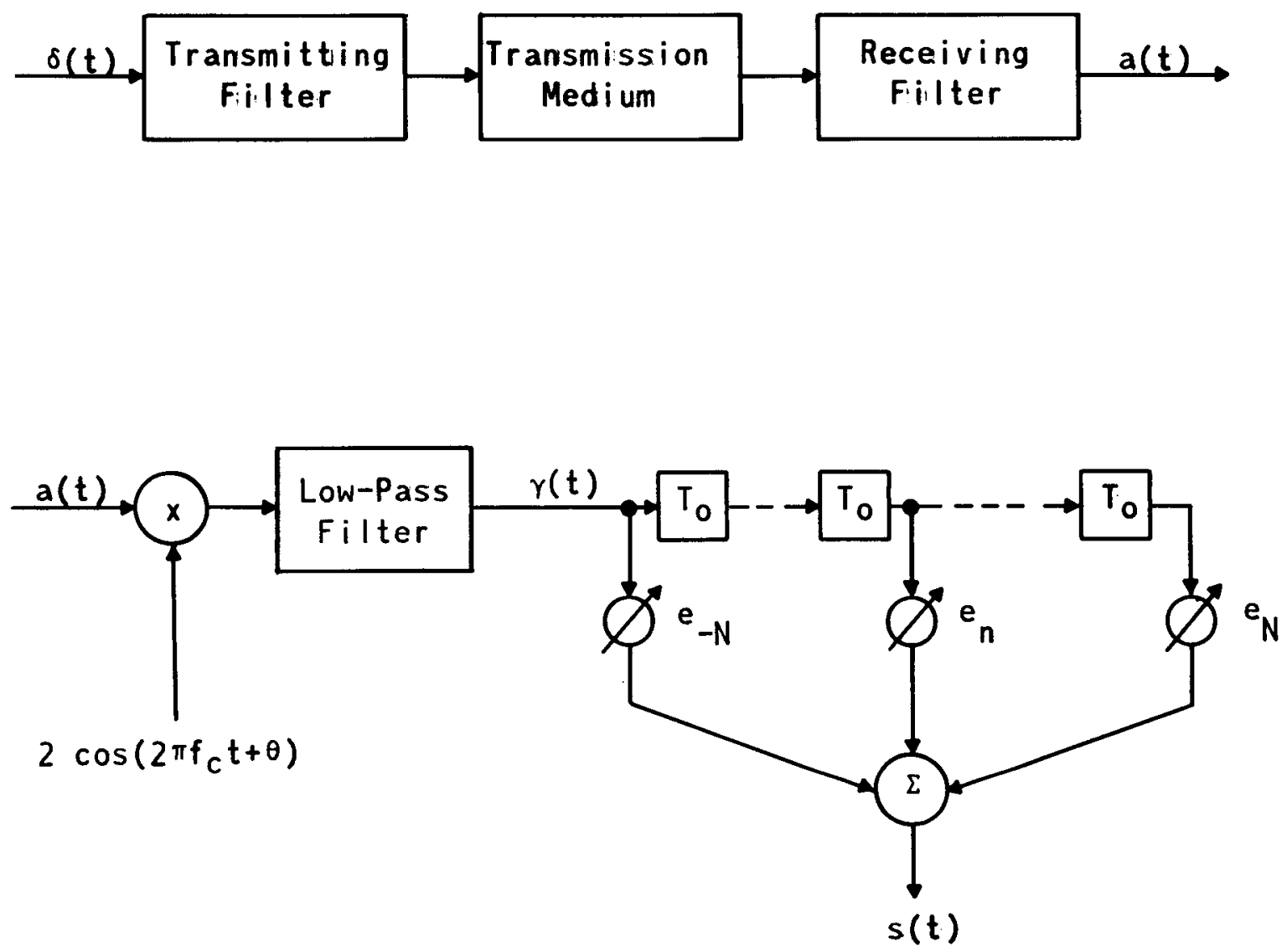

Figure 1. An amplitude modulation system with coherent detection and transversal filter equalization. 


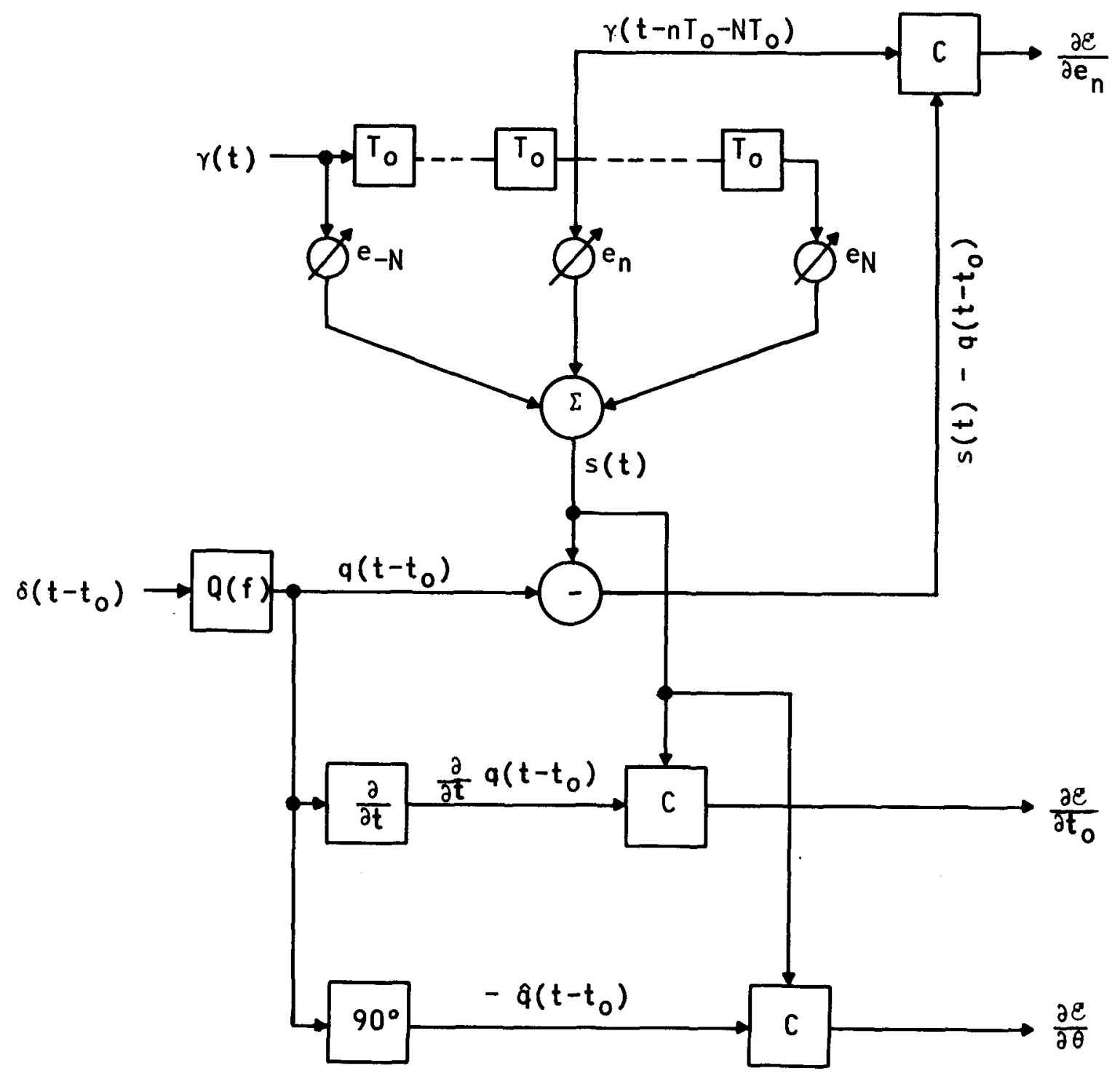

Phase Shifter

Figure 2. Block diagram of the joint method ( $C$ in the block denotes correlator). 